\title{
Single-Mode Fiber Refractive Index Sensor Based on Core-Offset Attenuators
}

\author{
Zhaobing Tian, Graduate Student Member, IEEE, Scott S-H. Yam, Member, IEEE, and Hans-Peter Loock
}

\begin{abstract}
Mach-Zehnder and Michelson interferometers using core-offset attenuators were demonstrated. As the relative offset direction of the two attenuators in the Mach-Zehnder interferometer can significantly affect the extinction ratio of the interference pattern, single core-offset attenuator-based sensors appear more robust and repeatable. A novel fiber Michelson interferometer refractive index (RI) sensor was subsequently realized by a single core-offset attenuator and a layer of $\sim 500-\mathrm{nm}$ gold coating. The device had a minimum insertion loss of $0.01 \mathrm{~dB}$ and maximum extinction ratio over $9 \mathrm{~dB}$. The sensitivity $(0.333 \mathrm{~nm})$ of the new sensor to its surrounding RI change $(0.01)$ was found to be comparable to that $(0.252 \mathrm{~nm})$ of an identical long period gratings pair Mach-Zehnder interferometric sensor, and its ease of fabrication makes it a low-cost alternative to existing sensing applications.
\end{abstract}

Index Terms-Fiber attenuator, Mach-Zehnder interferometer, Michelson interferometer, refractive index (RI) sensor.

\section{INTRODUCTION}

$\mathbf{O}$ PTICAL fiber interferometer sensors have been intensively studied to monitor quantities such as temperature, stress, gas phase concentrations, or refractive index (RI) change in solutions due to their compact footprints and ability for high resolution detection. One way to realize a sensor in a single-mode fiber (SMF), where light is confined in the core, is by long-period gratings (LPGs) [1], [2], which couple a fraction of the guided light from core to cladding (or reverse). Mach-Zehnder [3] interferometers and Michelson [4] interferometers have been constructed subsequently from LPG to further enhance measurement sensitivity. In another approach, light is coupled from a single-core fiber to a multicore fiber, thereby generating two different modes in two individual cores for Michelson interferometry [5]. However, in terms of RI measurement, these approaches have their respective drawbacks. For example, LPG-type sensors are responsive to a large range of RI, but require precise (and often expensive) photolithograhic alignment equipment and amplitude masks.

Manuscript received March 4, 2008; revised April 17, 2008. This work was supported by the Natural Science and Engineering Council of Canada (RGPIN 311817-06), Canadian Institute for Photonic Innovations and Photonics Research Ontario.

Z. Tian is with the Department of Physics, Engineering Physics, and Astronomy, Queen's University, Kingston, ON, K7L 3N6, Canada (e-mail:tianzhaobing@gmail.com).

S. S-H. Yam is with the Department of Electrical and Computer Engineering, Queen's University, Kingston, ON, K7L 3N6, Canada (e-mail: scott.yam@queensu.ca).

H.-P. Loock is with the Department of Chemistry, Queen's University, Kingston, ON, K7L 3N6, Canada (e-mail: hploock@chem.queensu.ca).

Color versions of one or more of the figures in this letter are available online at http://ieeexplore.ieee.org.

Digital Object Identifier 10.1109/LPT.2008.926832

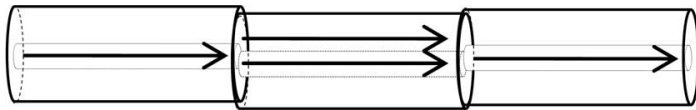

a. Mach-Zehnder Interferometer
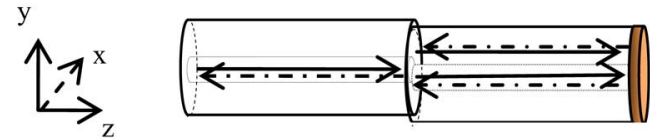

b. Michelson Interferometer

Fig. 1. Structure of core-offset attenuator-type interferometer

Coupling from a single-core to a multicore fiber requires specialty fiber and the two core-confined modes are not sensitive to the outer environment's RI variation. Recently, novel SMF Mach-Zehnder [6] and Michelson [7] interferometers based on abrupt tapers were demonstrated to have sensitivity similar to LPG-type interferometers at a fraction of the fabrication cost. In this letter, we report a new SMF sensor based on core-offset attenuators. The novel device has similar sensitivity as that of LPG or abrupt taper-type sensor, while further reducing fabrication complexity.

\section{PRINCIPLE}

Optical attenuators have been implemented widely [8] in optical communication systems for different functions, such as receiver input power control, wavelength balancing, and power equalization in wavelength-division-multiplexing systems. One common design achieves fixed attenuation by offsetting two SMF cores by several micrometers. Light from the transmission fiber is split into two paths, with a fraction of light remaining in the core of the receiver fiber, while the remainder is transferred to the cladding and subsequently propagates as cladding modes. Due to the attenuation at the cladding-coating interface, the cladding modes cannot propagate over a long distance. However, if another core-offset is introduced only several centimeters after the first one, light in the cladding can be coupled back to the core with ignorable loss [Fig. 1(a)]. Due to the phase difference of the cladding and core modes, a Mach-Zehnder interferometer is realized by the second offset. In a related approach, a gold layer is deposited on the fiber end facet only several centimeters after the offset section [Fig. 1(b)], and light propagating in the cladding is reflected and coupled back into the core (dashed line). In that case, a Michelson interferometer is realized when the reflected light propagates twice through the same SMF offset. When light emitted by a broadband source (BBS) propagates through either the Mach-Zehnder or Michelson interferometer, interference patterns may be recorded by an optical spectrum analyzer (OSA). If the RI of the environment sur- 


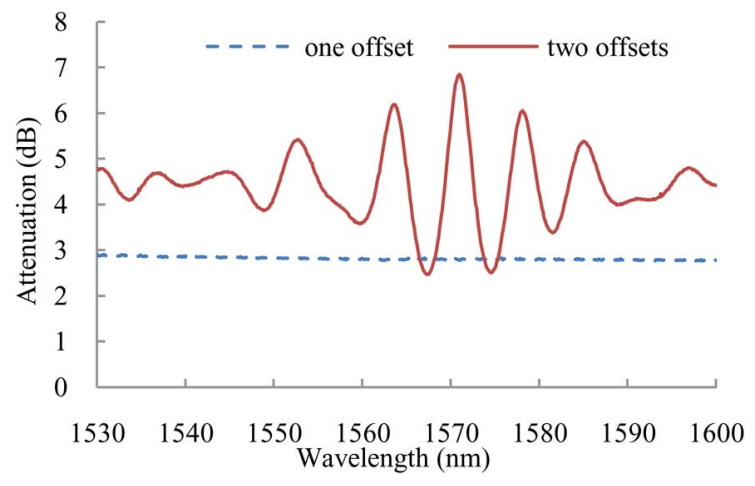

Fig. 2. Spectrum of Mach-Zehnder interferometer $(80 \mathrm{~mm})$.

rounding the SMF increases, for example, by submersing the fiber in water, the effective RI of the cladding mode increases, while that of the core mode stays almost constant. Therefore, the effective RI difference between the core and cladding modes decreases and the interference pattern recorded by the OSA shifts to a shorter wavelength.

\section{EXPERIMENT}

To characterize the interference response, light from a BBS (JDS Uniphase, 1520-1610 nm) was launched from an SMF input and the transmission spectrum was recorded by an OSA (ANDO AQ6317B) in the decibel domain. The attenuation spectrum of the devices was obtained by subtracting the source's spectrum from the device's transmission spectrum. A fusion splicer (Ericsson FSU 995FA) with a built-in attenuator program was used to fabricate the $3-\mathrm{dB}$ attenuators. For the Mach-Zehnder interferometer, first a 3-dB attenuator was made and the attenuation spectrum was recorded. A second $3-\mathrm{dB}$ attenuator was then made $80 \mathrm{~mm}$ away from the first one, and the fiber coating between the two attenuators was stripped off. The device was mounted and straightened on positioning stages, while the attenuation spectrum was recorded. As shown in Fig. 2, the spectrum of a single 3-dB attenuator is flat in the whole wavelength window (dashed line). After the second offset was made, an interference pattern (solid line) was observed, exhibiting a $4.8-\mathrm{dB}$ extinction ratio and $2.8-\mathrm{dB}$ minimum insertion loss. Interestingly, the minimum attenuation of the interferometer is less than that of one 3-dB attenuator, observed at 1566 and $1574 \mathrm{~nm}$ in Fig. 2. At those wavelengths, the core mode and cladding mode interfere constructively. Fig. 2 also shows that the interference is wavelength-dependent due to the existence of multiple copropagating cladding modes.

Compared with the result of the abrupt-taper-based Mach-Zehnder interferometer [6], which has an extinction ratio of more than $23 \mathrm{~dB}$ and a minimum loss of $3 \mathrm{~dB}$, the extinction ratio is considerably smaller. The difference can be due to the relative offset direction between the two attenuators, as the taper has axial symmetry.

To investigate the relationship between the relative offset direction and the Mach-Zehnder interference pattern, the following experiment was conducted. An SMF pigtail, which was spliced onto a $60-\mathrm{mm}$ bare SMF segment by $3-\mathrm{dB}$ offset facet attenuator on the tip, was mounted on the translation

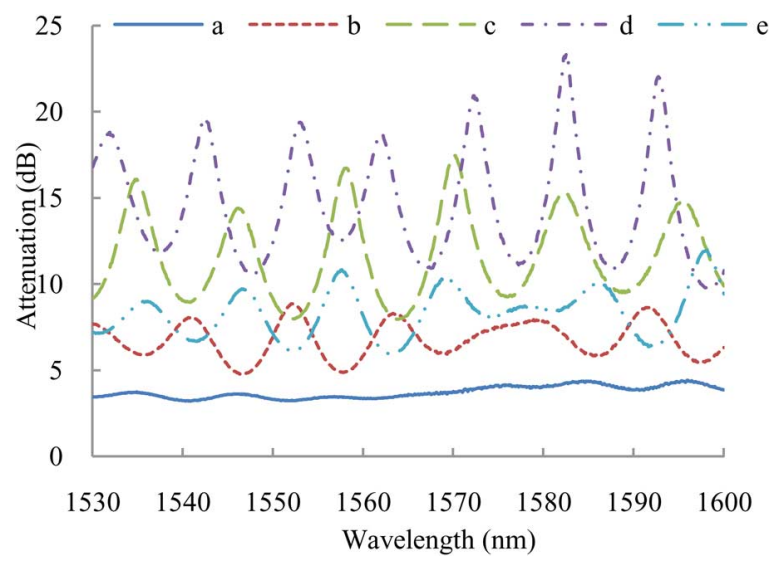

Fig. 3. Mach-Zehnder interferometer $(60 \mathrm{~mm})$ spectrum of (a) "zero" position, (b) 7- $\mu \mathrm{m}$ offset along $\mathrm{x}$-axis, (c) $-7-\mu \mathrm{m}$ offset along $\mathrm{x}$-axis, (d) 7- $\mu \mathrm{m}$ offset along $\mathrm{y}$ - axis, and (e) $-7-\mu \mathrm{m}$ offset along $\mathrm{y}$ - axis.

stage (Thorlabs NanoMax TS 6-Axis) so that it can be aligned with respect to a second SMF pigtail. The two fiber ends were aligned with the help of a microscope (stereozoom trinocular microscope coupled to Nikon charged coupled device). Optimal core overlap, called the "zero position" below, was achieved when the claddings of the two fibers was aligned. The fiber axis was defined as z-axis in the subsequent experiment. The spectrum at this "zero" position was recorded [Fig. 3(a)], and the fiber pigtails were displaced by $7 \mu \mathrm{m}$ along the $\mathrm{x}$-axis [Fig. 3(b)]. Similarly, the spectrum shown in Fig. 3(c) was obtained by displacing the fibers by $-7 \mu \mathrm{m}$ along the $\mathrm{x}$-axis, whereas Fig. 3(d) and (e) shows spectra following displacement by +7 and $-7 \mu \mathrm{m}$ along the $\mathrm{y}$-axis, respectively. The spectrum obtained at the zero position shows no interference pattern [Fig. 3(a)] and corresponds to a constant attenuation of $3 \mathrm{~dB}$ over the entire wavelength region of interest. This was expected considering that Fig. 2 (dashed line) also shows the similar spectrum for one offset attenuator. The large differences observed for loss and extinction ratios of curves (b)-(e) show that the relative offset direction between the two attenuators can affect the interferometer performance greatly. For curves (b) and (c), and curves (e) and (d), the interference patterns were opposite, in agreement with the Mach-Zehnder interferometer theory, that predicts that one output port is constructive interference, the other one must be destructive interference. For the fusion splicer that lacks functionalities for polarization-maintaining fibers, it is difficult to control the relative offset direction between the two attenuators, so practical application of the Mach-Zehnder interferometer using core-offset as a sensor is limited by high insertion loss and low extinction ratio.

In the case of the Michelson interferometer, the direction of the fiber core offset is of no concern since only a single attenuator is used. As shown in Fig. 1(b), a layer of $\sim 500$-nm gold was sputter coated on the fiber end facet. Again a 3-dB offset attenuator was made using the fusion splicer. The length between the mirror and attenuator section was $38 \mathrm{~mm}$. The spectrum of the single-offset Michelson interferometer exhibits and extinction ratio of $9 \mathrm{~dB}$ and minimum loss of $0.01 \mathrm{~dB}$ (Fig. 4). The $\sim 15$-nm bandwidth is larger than that in Figs. 2 and 3 considering the effect of the interferometer length. This is due to the 


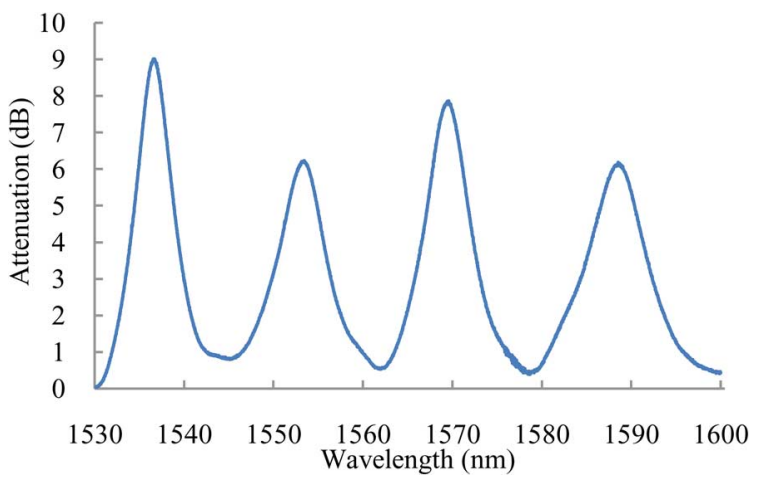

Fig. 4. Spectrum of Michelson interferometer.

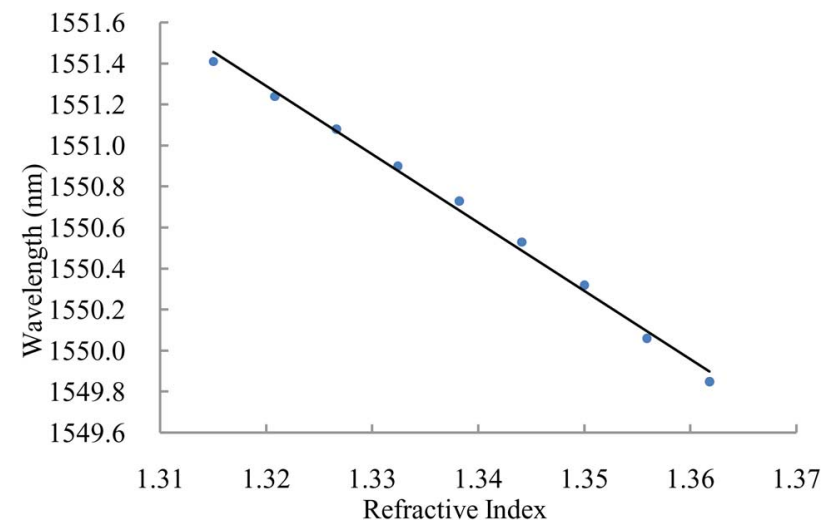

Fig. 5. Maximum attenuation wavelength shift of Michelson interferometer due to solutions of different refractive indexes.

fact that the cladding mode's order in Fig. 4 is smaller than that of Figs. 2 and 3, which decreases the effective RI difference between core and cladding mode and results a larger bandwidth. The Michelson interferometer was tested as a sensor for ambient RI change. An adjustable stage was placed below the fiber to support the test sample solution glass cup. The sensor was then immersed in the RI standard solution while the output spectrum was recorded by OSA. After the measurement, the device was cleaned with distilled water and compressed air. A different RI standard was then applied, and the procedure was repeated. Nine dimethyl sulfoxide solutions with different concentrations $(0.0 \%, 4.0 \%, 8.0 \%, 12.0 \%, 16.0 \%, 20.0 \%, 24.0 \%, 28.0 \%$, and $32.0 \%)$ were used in this experiment. The corresponding RIs (calculated from Fresnel reflection using water as reference at $25^{\circ} \mathrm{C}$ for the 1550 -nm wavelength) are $1.315,1.3208,1.3266$, $1.3324,1.3382,1.3441,1.35,1.3559$, and 1.3618 , respectively. Fig. 5 shows the attenuation maxima wavelength shift with the change of the RI of the sample. For a 0.01 RI change, a $0.333-n m$ shift was observed. The response of the new sensor is comparable with that of an LPG pair sensor $(0.259 \mathrm{~nm})$ [3], which interacted with a solution over a length of $62 \mathrm{~mm}$. On the other hand, it may be more meaningful to consider that the total interaction length of the Michelson interferometer is twice that of the 38-mm arm exposed to the sample. With this correction, we found that the sensitivity of the LPG interferometric sensor (4.2 $\mathrm{pm} / \mathrm{mm}$ for $\Delta n=0.01$ ) and the present Michelson inter- ferometer $(4.4 \mathrm{pm} / \mathrm{mm})$ are very close. We note that the fabrication of one attenuator structure is simpler compared to that of the LPG pair and manufacture of one 3-dB attenuator normally takes less than $1 \mathrm{~min}$. For RI in the range of 1.3-1.4, which is the typical range of protein analytes, the sensitivity of a sensor using a single core-offset attenuator Michelson interferometer should be uniform. Increasing the interaction length with the sample between the attenuator and mirror linearly increases the sensitivity. Also the sensitivity is expected to increase with cladding mode order and with decreasing fiber cladding diameter since both increase the phase difference between the core and the cladding modes for the same RI change.

\section{CONCLUSION}

Two new types of fiber-optic interferometers (Mach-Zehnder and Michelson) using core-offset attenuators have been demonstrated. It is found that relative offset directions between the two attenuators needed to construct a Mach-Zehnder interferometer can affect the interference performance greatly. By comparison, the Michelson interferometer used only a single core-offset joint. The minimum loss of the Michelson interferometer was $0.01 \mathrm{~dB}$, while the maximum extinction ratio was more than $9 \mathrm{~dB}$. The Michelson interferometer was tested as an RI sensor, and the sensitivity was found to be comparable to that of a similar LPG-type sensor. Interferometers based on core-offset attenuators, therefore, combine high sensitivity to RI changes with very low cost of fabrication and a response over a wide wavelength range.

\section{ACKNOWLEDGMENT}

The authors would like to thank the Canadian Microelectronics Corporation for their assistance with the experiments. Some measurements were made with equipment from the National Microelectronics and Photonics Testing Collaboratory.

\section{REFERENCES}

[1] H. J. Patrick, A. D. Kersey, and F. Bucholtz, “Analysis of the response of long period fiber gratings to external index of refraction," J. Lightw. Technol., vol. 16, no. 9, pp. 1606-1612, Sep. 1998.

[2] K. S. Chiang, Y. Liu, M. N. Ng, and X. Dong, "Analysis of etched long-period fiber grating and its response to external refractive index," Electron. Lett., vol. 36, pp. 966-967, Apr. 2000.

[3] J. F. Ding, A. P. Zhang, L. Y. Shao, J. H. Yan, and S. He, "Fiber-taper seeded long-period grating pair as a highly sensitive refractive-index sensor," IEEE Photon. Technol. Lett., vol. 17, no. 6, pp. 1247-1249, Jun. 2005.

[4] P. L. Swart, "Long-period grating Michelson refractometric sensor," Meas. Sci. Technol., vol. 15, no. 8, pp. 1576-1580, Aug. 1, 2004.

[5] L. Yuan, J. Yang, Z. Liu, and J. Sun, "In-fiber integrated Michelson interferometer," Opt. Lett., vol. 13, pp. 2692-2694, 2006.

[6] Z. Tian, S. S.-H. Yam, J. Barnes, W. Bock, P. Greig, J. M. Fraser, H. P. Loock, and R. D. Oleschuk, "Refractive index sensing with Mach-Zehnder interferometer based on concatenating two single-mode fiber tapers," IEEE Photon. Technol. Lett., vol. 20, no. 8, pp. 626-628, Apr. 2008.

[7] Z. Tian, S. S.-H. Yam, and H. P. Loock, "Refractive index sensor based on an abrupt taper Michelson interferometer in a single mode fiber," Opt. Lett., vol. 33, pp. 1105-1107, 2008.

[8] D. K. Mynbaev and L. L. Scheiner, Fiber-Optics Communications Technology. New York: Pearson Education, Inc., 2002, pp. 634-1634. 